\title{
Internet y el ejercicio de los derechos de los accionistas en las empresas: un análisis empírico
}

\author{
María del Mar Alonso Almeida \\ Universidad Autónoma de Madrid, España / mar.alonso@uam.es.
}

Recibido: 9 de septiembre de 2008

Aceptado: 22 de abril de 2009

\section{RESUMEN}

La globalización de la economía y los escándalos financieros en algunas empresas han hecho que la información sobre el funcionamiento de las empresas sea un asunto crítico para la confianza y para el buen funcionamiento del mercado de capitales. Este hecho ha provocado la proliferación de códigos de buen gobierno relativos a las prácticas que las empresas deben seguir en el seno de la dirección.

El presente trabajo se analizará si la tecnología de Internet puede contribuir al fortalecimiento de la relación entre accionistas y empresas, en el ejercicio de los derechos del accionista en la empresa.

Palabras clave: accionistas, internet, gobierno corporativo electrónico, participación electrónica, código de buen gobierno.

\section{ABSTRACT}

The globalization and some financial scandals have turned the corporate governance in a critical issue for the firms and the capital markets. Due to this situation, the good governance codes about best practices in the boards and the directorship have spread.

This article analyzes if internet can strengthen the relationship between shareholders and firms and increases the participation in the making decision into the firm.

Keywords: shareholders, internet, Internet, electronic corporate governance, electronic participation, good governance code. 


\section{INTRODUCCIÓN}

Las actuales formas de gobierno corporativo se han visto cuestionadas debido a los escándalos que han rodeado a algunas organizaciones empresariales. Esto ha provocado que se levanten algunas corrientes doctrinales, como la teoría stakeholder, que piden mayor transparencia, traducida en mayor información, mayor accesibilidad y publicidad de la información y mayor participación de todos los implicados en la organización.

Los nuevos enfoques sobre la teoría de la organización y sobre el cambio de comportamiento de gobierno organizativo, tal y como esquematiza Zingales (2000) y resume Bueno (2004), se fundamentan a partir de la evolución de las cuatro concepciones siguientes:

a) La observación de la organización como un nexo de contratos explícitos valorizados por los mercados financieros y basados en las perspectivas de los costes de transacción, derechos de propiedad y relación de agencia (Alchian y Demsetz, 1972; Jensen y Meckling, 1976; Fama y Jensen, 1983; Willianson, 1985), propios del enfoque contractual clásico y de la teoría actual.

b) El análisis de la organización como nexo de contratos explícitos e implícitos en el que cobra importancia el capital relacional, consecuente con la existencia de contratos relacionales (Rajan y Zingales, 1998; Backer et al., 2002; Rodríguez Fernández, 2003), enfoque característico de la teoría institucional.

c) El enfoque de la empresa como una colección de oportunidades de crecimiento, basados en los activos o recursos críticos y en las capacidades para generar las citadas oportunidades (Wernerfelt, 1984; Teece, Shuen y Pisano, 1997).

d) La visión de la empresa como colección de activos tangibles e intangibles derivados de cada categoría de los contratos que se integran en la organización (Grossman y Hart, 1986; Nonaka y Takeuchi, 1995; Grant, 1996), característico del en- 
foque actual basado en conocimiento (Spender, 1996; Grandori, 2004).

Esta evolución culmina en la necesidad de definir un nuevo modelo de empresa basado en el conocimiento y en las relaciones con el resto de los partícipes y agentes sociales que configuran lo que se viene llamando "organización nexo o red" y que configura el capital relacional de la empresa (Bueno, 2004; Foss, 2005). Este nuevo modelo de empresa requiere una nueva estrategia de gobierno, y por tanto un nuevo comportamiento del gobierno de la empresa para su desarrollo (Alonso Almeida, 2007).

Esta estrategia parte del llamado movimiento de reforma en relación al "buen gobierno corporativo", materializado en unos códigos de prácticas de buen gobierno y legislación de obligado cumplimiento que recomiendan el uso de una tecnología de comunicación universal, como es Internet, para proporcionar confianza al mercado (Jones, 1995).

Dichos códigos, de otra parte, han sido consecuencia lógica de la construcción de un marco teórico y de unas prácticas concordantes con la época de la economía industrial. Éstos han cambiado en los último años, como consecuencia del cambio profundo en los procesos económicos derivado de la "era de los intangibles" y de la Sociedad de la Información, todo lo cual ha llevado a situaciones no explicadas por aquél marco teórico, y a nuevas prácticas o formas de conocer y de hacer (Saudagaran y Diga, 1997; Waltz, 1999).

Desde finales de la década de los años noventa, las compañías más comprometidas con la transparencia hacen uso de la tecnología de Internet para mejorar la comunicación con sus inversores y para mejorar su imagen. Además, los organismos reguladores de los principales mercados financieros han desarrollado normativas que especifiquen la utilización de la web como elemento para fomentar la transparencia.

En este trabajo se intenta conceptuar el significado del gobierno corporativo electrónico, describir las principales herramientas que Internet pone a disposición de las empresas para el desarrollo de los mecanismos de gobierno electrónico y evaluar la situación en el mercado español. Cabe de más decir que Internet debe ser uno más 
de los canales de comunicación que la empresa ponga a disposición de los accionistas y no el único. De no ser así, en lugar de beneficiar al accionista se le terminaría perjudicando, ya que en la actualidad no todos los accionistas acceden a la información de las empresas a través de Internet.

\section{EL GOBIERNO CORPORATIVO ELECTRÓNICO}

El gobierno corporativo electrónico implica el uso de la tecnología de Internet para mejorar la confianza entre las empresas y los grupos de interés. Pero para que éste obtenga el resultado esperado debe haber participación; debe producirse lo que Handy (1995) denomina la aceptación de la tecnología.

Davis (1989) investigó sobre las causas que motivaban a los empleados a adoptar una nueva tecnología en su trabajo y llegó a la conclusión que principalmente se debe a dos causas: 1) percepción de utilidad y 2) percepción de facilidad de uso. Sus investigaciones le condujeron a enunciar el Modelo de Aceptación de la Tecnología (Technology Acceptance Model), conocido por sus siglas en inglés, TAM. Este modelo ha sido probado y validado en diversas investigaciones, tanto con distintas herramientas de trabajo tecnológicas (Pavlou, 2003), como en Internet (Dahlberg et al., 2003). El TAM se basa en la teoría de que las creencias sobre algo influyen en las intenciones, y las intenciones, sobre la acción. Siguiendo este razonamiento, para que se acepte la tecnología de Internet por los accionistas, ésta debe percibirse como útil y fácil de usar.

El modelo de la Difusión de la Innovación (DOI por sus siglas en inglés), enunciado por Rogers (1996), es otro modelo de investigación que explica la aceptación de la tecnología. Este modelo se basa en cuatro características: 1) complejidad definida como el grado en que la tecnología es fácil de entender y usar; 2) compatibilidad o grado en que la tecnología es compatible con la creencia, los valores y la experiencia de los que van a usarla; 3) utilidad o capacidad de uso y 4) observabilidad o el grado en que los resultados son visibles. De estas cuatro características, la doctrina sobre la inteligencia artificial concluye que las más relevantes para la adopción de la 
nueva tecnología son la complejidad y la compatibilidad (Hoffman y Novak, 1996).

Otros autores presentan otros factores como la imagen de la empresa. Las investigaciones realizadas que utilizan la tecnología de Internet demuestran que la imagen de la empresa que usa la tecnología proporciona razones para su uso. Asimismo, se percibe una ventaja por el uso de la tecnología (Bolton y Katok, 2004). De acuerdo con estos modelos, se puede decir que los accionistas estarán más dispuestos a usar la tecnología de Internet si perciben facilidad de uso, utilidad y la ventaja que obtienen por su uso.

Los pequeños accionistas conforman el colectivo que más acusa la rapidez y volatilidad de los mercados de valores. Por ello se hace cada vez más importante que las sociedades anónimas cotizadas se esfuercen por ofrecerles toda la información necesaria que les permitan adoptar sus decisiones de inversión en igualdad de condiciones con los inversores institucionales y los grandes accionistas.

En línea con este razonamiento, en los últimos años se han producido en Europa diversos análisis empíricos sobre la información, principalmente financiera, que las principales compañías europeas distribuían a través de Internet.

En ese sentido, Lymer (1998) analiza las 50 mayores empresas de Reino Unido. Craver y Marston (1999) avanzan en esta dirección analizando 206 empresas del índice FTSE 100 y las compañías con las mayores capitalizaciones, de acuerdo con la información publicada en el periódico Financial Times. En Alemania, Marston y Polei (2004) examinan la información voluntaria y la información relativa a los inversores en las principales empresas alemanas. Lymer y Tallberg (1997) analizan la información de las empresas que cotizan en la Bolsa de Helsinki. Hedlin (1999) estudia las 60 compañías suecas que cotizan en el incide de la Bolsa de Estocolmo. En España, también se han desarrollado algunos análisis empíricos en este campo. Así, Gowthorpe y Amat (1998) analizaron las 379 empresas que cotizan en la Bolsa de Madrid. Larrán y Giner (2002) realizaron un estudio sobre la información presentada en Internet por las 144 empresas que cotizan en el mercado continuo, al igual que Gandia y Andrés (2005). Otros análisis empíricos han analizado 
los principales índices europeos. Bonsón et al. (2000) analizaron los informes digitales de las 50 empresas que componen el índice Dow Jones Eurostoxx 50. Más tarde Bonsón y Escobar (2002) analizaron la información financiera digital de las 300 mayores empresas de la Unión Europea. Posteriormente, han analizado con dos objetivos la información que las empresas de los países de Europa del Este que se están incorporado en los últimos años a la Unión Europea proporcionaban a través de Internet. El primer objetivo es determinar la distancia existente entre la información que actualmente proporcionan y la que se exige, según las directivas europeas. El segundo es analizar empíricamente los factores que explican el comportamiento diferente de las empresas en la relación con los inversores y accionistas (Bonsón y Escobar, 2006).

Puesto que la información proporcionada a través de la página web se configura como una de las herramientas principales para el fortalecimiento de la relación entre empresa y accionistas, se va a analizar la situación en el mercado español cotizado, principalmente su utilización y resultados.

Hay que tener en cuenta que los problemas existentes entre las sociedades y sus accionistas no se producen en las sociedades cerradas con un número de accionistas reducido, los cuales se conocen entre sí y están unidos por vínculos familiares o de confianza más o menos estrechos, sino en las sociedades abiertas o cotizadas con un número elevado de accionistas con diferentes inversiones e intereses, y donde las nuevas tecnologías pueden atenuar el efecto de la apatía del accionista. En las sociedades cotizadas es donde los medios tecnológicos de participación pueden hacer que el ejercicio de los derechos políticos del accionista deje de resultar una carga antieconómica para los pequeños accionistas. Puesto que la información proporcionada a través de la página web se configura como una de las herramientas principales para el fortalecimiento de la relación entre empresa y accionistas, el análisis empírico se va a centrar en el desarrollo del gobierno electrónico a partir de las recomendaciones de los códigos de buen gobierno y del uso de la tecnología (Belanger y Hiller, 2005).

Hay que considerar una serie de factores que la doctrina ha puesto de manifiesto. En primer lugar, las empresas más grandes 
tienen un mayor número de accionistas e inversores pendientes de su evolución; por eso necesitan ofrecer información que contribuya a la comprensión de las empresas a los agentes interesados (Marston y Polei, 2004). En segundo lugar, las grandes compañías son más visibles y, como consecuencia, tienen más costes políticos (Watts y Zimmermann, 1978). En tercer lugar, desde el punto de vista de la teoría de la agencia, el incremento de información puede reducir los costes de agencia y las asimetrías de información y, consecuentemente, incrementar la confianza en la empresa (Jensen y Merckling, 1976; Hossain et al., 1995, Oyelere et al., 2003). Como resultado, las empresas de mayor tamaño tienen fuertes incentivos para intentar incrementar la comunicación con sus accionistas a través de Internet. La teoría enunciada lleva a plantear la siguiente hipótesis de estudio:

H1: El tamaño de la empresa está relacionada con su disposición de herramientas en Internet para relacionarse con los accionistas.

La mayoría de los estudios realizados hasta ahora se centran en analizar la información financiera y parte de la información sobre gobierno corporativo que las empresas ponen a disposición de los terceros en su página web. Esta investigación pretende ir más allá y analizar, no si las empresas cumplen con la legalidad y son transparentes en su gobierno, sino si a través de la información, canales de comunicación, otras funcionalidades y características propias de la tecnología, las empresas pueden ayudar en el ejercicio de los derechos sociales a los accionistas minoritarios.

La Junta de accionistas es el medio por excelencia donde los accionistas pueden ejercer sus derechos como propietarios. La aplicación de la tecnología de Internet en la Junta de accionistas se puede agrupar en tres esquemas:

a. Voto electrónico. Entendiendo como tal, el ejercicio del voto del accionista que no está presente físicamente en la Junta. Los votos pueden ser recogidos con anterioridad a la Junta o pueden ser emitidos de forma simultánea a 
la votación virtual, lo que se denomina "participación virtual" (Muñoz Paredes, 2004). Los accionistas que voten a distancia se contarán, a efectos de constitución de la Junta, como presentes. Este método puede satisfacer los intereses de los accionistas que quieran participar en las Juntas. Favorece la comunicación y el compromiso con la empresa.

b. Participación a distancia por sedes interconectadas con la sede principal donde se lleva a cabo físicamente la junta, y conectadas de forma virtual por Internet desde su casa, oficina u otro lugar, no controlado por la empresa.

c. Junta virtual. Cuando todos los accionistas están conectados de forma virtual, sin reunión física. Los asistentes podrán ejercitar sus derechos de solicitar la palabra, hacer preguntas, derecho de réplica y contrarréplica.

La aplicación de estas formas de participación entrañan dificultades que hay que tener en cuenta para garantizar el éxito y la confianza de los accionistas (Muñoz Paredes, 2004). Por eso se debe habilitar un sistema seguro basado en firma electrónica o similar que garantice la correcta identificación del accionista o su representante.

Como consecuencia, el uso de herramientas de Internet para la participación en la vida social de la empresa nos lleva a plantear la siguiente hipótesis de estudio:

H2: El voto electrónico incrementa la participación del accionista en la vida social de la empresa.

\section{ANÁLISIS EMPÍRICO}

El universo que nos va a permitir contrastar las hipótesis planteadas en el apartado anterior de esta investigación son las empresas que cotizan en el mercado continuo en España. De este universo, se ha seleccionado una población compuesta por las empresas que tienen una capitalización mayor de 200 millones de euros, en total 95 empresas. 
En esta investigación, salvo algún dato concreto o aclaración adicional que se ha necesitado recabar de la empresa (en concreto del Departamento de relación con los inversores), la información se ha obtenido de fuentes secundarias. Las fuentes de datos utilizadas han sido las páginas web de las empresas objeto de la muestra, las páginas web de las Bolsas de Madrid, Barcelona y Bilbao, así como las Oficinas de estudios de dichas Bolsas y las Oficinas del accionista de las empresas de la muestra.

La variable dependiente es la existencia de voto electrónico (EVOTO) en la página web de la empresa y las variables independientes, las variables analizadas en los contrastes. La variable E-VOTO es una variable dicotómica que mide si la empresa pone a disposición de los accionistas el voto electrónico o no. En el caso de que la empresa no utilice voto electrónico en las Juntas de Accionistas, el valor es 0 . Las variables independientes, son las que dan respuesta a las hipótesis planteadas. Entre las variables independientes, se incluye la variable TAMAÑO. Esta variable puede ser cuantificada por diversas medidas. En este caso se va a utilizar la capitalización de las compañías al cierre del ejercicio. Se usará el valor del logaritmo neperiano de este valor.

La variable ACCIONISTAS es una variable subrogada que se mide con el porcentaje del capital flotante de cada empresa al final del ejercicio. El valor se toma de los informes emitidos por la CNMV sobre distribución de la propiedad de las empresas cotizadas.

La variable E-PARTICIPACION mide si ha habido voto electrónico en las empresas o no. Es una variable numérica que indica el número de votos electrónicos habido en la Junta. En concreto, el porcentaje de votos electrónicos sobre el total de votos. Si la empresa no dispone de esta modalidad de voto el valor es 0 . El dato se ha tomado de la página web de la empresa y de las entrevistas realizadas a las Oficinas del accionista.

Los resultados obtenidos nos muestran que un 33,7 por ciento de la muestra utiliza el voto electrónico para estimular la participación de su accionariado disperso, lo que representa 32 empresas. Por capitalización se distribuye según la tabla 3: 
TABla 3. Empresas que Utilizan El Voto electrónico POR CAPITALIZACIÓN

\begin{tabular}{lc} 
Capitalización & No empresas \\
\hline IBEX & 15 \\
$>1000$ millones de $€$ & 8 \\
$<1000$ millones $y>500$ millones $€$ & 4 \\
$<500$ millones $y>200$ millones $€$ & 5 \\
\hline
\end{tabular}

Como se puede observar, las empresas más grandes pertenecientes al IBEX35 son las empresas que principalmente ponen a disposición de los accionistas esta herramienta de participación: un 40\% de las empresas pertenecientes a este grupo.

El análisis de correlación del coeficiente de Pearson nos muestra los siguientes resultados (tabla 4 ):

Tabla 4. Resultados análisis Univariante

\begin{tabular}{lrccc} 
Variable & E-VOTO & Accionistas & Tamaño & E-Participación \\
\hline E-VOTO & 1 & & & \\
ACCIONISTAS & .071 & 1 & & \\
TAMAÑO & $.269\left(^{* *}\right)$ & $\left..269^{* *}\right)$ & 1 & 1 \\
E-PARTICIPACIÓN & $.49\left(^{* *}\right)$ & .096 & $.392\left(^{* *}\right)$ & 1 \\
\hline
\end{tabular}

Los resultados del análisis univariante muestran una relación positiva entre: el tamaño de la empresa, la participación y el voto electrónico. Estos resultados demuestran que las empresas de mayor tamaño tienen una mayor participación de sus accionistas en la vida social por Internet. Sin embargo, no parece que exista relación entre los accionistas y el voto electrónico.

Con el fin de obtener las variables que en mayor medida contribuyan a explicar la variabilidad del modelo, se ha utilizado como procedimiento de regresión múltiple, el método de regresión de etapas sucesivas (stepwise regression). Este método se trata de un método de regresión por etapas "hacia delante", en el que la entrada y salida de las variables en la ecuación de regresión se realiza a partir de los valores del estadístico t-Student (Stevens, 1996). En cada paso se calcula el coeficiente estandarizado para cada variable, en el 
supuesto que la misma entrase en el modelo en el próximo paso, así como el valor del estadístico t y el nivel de significación. De esta forma se evita la colinealidad y se seleccionan las variables independientes que más influyen en las hipótesis que se quieren contrastar. En este estudio se ha utilizado como criterio de entrada en la ecuación, un nivel de significación inferior a 0.05 y uno de salida superior a 0.1 . El análisis multivariante realizado confirma en parte los resultados obtenidos por el análisis univariante (ver tabla 5):

TABLA 5. Resultados ANÁLISIS REgResión

\begin{tabular}{lcccc} 
& Coeficiente & Std. error & $\mathbf{t}$ & Prob. \\
\hline \multicolumn{1}{c}{ (Constante) } & 5.097 & 5.204 & 0.979 & 0.330 \\
TAMAÑO & 3.475 & 0.371 & 9.336 & 0.000 \\
E-PARTICIPACIÓN & 9.439 & 1.539 & 6.131 & 0.000 \\
R2 & 0.642 & & Estadístico F & 39.902 \\
R2 Ajustada & 0.626 & & Prob. F & 0.000 \\
Error típico regresión & 5.757 & & Durbin-Watson & 1.827 \\
\hline
\end{tabular}

Dos son las variables que contribuyen en mayor medida a explicar los niveles de uso de Internet de los accionistas de las empresas. Estas variables son: a) el tamaño de la empresa, como se reconoce en buena parte de la evidencia empírica previa (Marston y Polei, 2004; Bonsón y Escobar, 2006) y b) la participación electrónica.

Desde el punto de vista estadístico, el valor del estadístico " $t$ " indica que las dos variables independientes seleccionadas por el modelo de regresión tienen una significación del 5\%. El coeficiente de determinación del modelo es de 0.642 , lo que significa que estas variables seleccionadas explican un $64.2 \%$ de la varianza del voto electrónico. Los estadísticos resumen, como el estadístico $\mathrm{F}$ y el Durbin-Watson, indican que no hay problemas de colinealidad, ni autocorrelación.

\section{RESULTADOS OBTENIDOS}

Según los resultados obtenidos por la estadística descriptiva, el análisis univariante y el análisis multivariante, se puede llegar a los siguientes resultados: 
H1: La disposición de herramientas en Internet por parte de las empresas para relacionarse con los accionistas se relaciona positivamente con su tamaño.

\section{Aceptada totalmente}

H2: El voto electrónico incrementa la participación del accionista en la vida social de la empresa.

\section{Aceptada totalmente}

Las empresas que tienen una mayor capitalización son las que más herramientas ponen a disposición de los accionistas para fortalecer su relación con éstos. Estas herramientas pueden llevar a conocer mejor a las empresas, a sus consejos de administración y a los directivos, lo que permite que se desarrolle una relación de confianza y compromiso activo con la empresa. De estas posibles herramientas que la empresa puede poner a disposición de los accionistas, el voto electrónico se configura como la que incrementa la participación de éstos en la vida social haciendo oír su voz.

La evidencia empírica demuestra que el hecho de que las empresas pongan a disposición de sus accionistas herramientas electrónicas para que participen desde cualquier lugar en donde se encuentren, ha favorecido el voto en las Juntas. Los datos recogidos muestran que en las empresas que cuentan con la opción de voto electrónico, esta opción ha sido utilizada en todos los casos por algún accionista. Esto sugiere que aunque en la actualidad no sea utilizado por gran parte de los accionistas, puede ser una herramienta que en el futuro ayude a disminuir la apatía de éste al permitir su participación desde cualquier lugar, con la consiguiente comodidad y ahorro de costes para el mismo.

\section{Conclusiones}

Las empresas se encuentran en un mercado de capitales globalizado. Las tecnologías de la información y las comunicaciones (TIC) se han convertido en agentes dinamizadores que potencian el valor de las organizaciones. Las voces críticas que se han levantado llevan a pensar que es necesaria una revisión de las prácticas de 
gobierno existentes y una nueva estrategia política que garantice la recuperación de la confianza de los accionistas e inversores. Esta nueva estrategia política debe incorporar un comportamiento ético de los agentes principales de las organizaciones para lograr una mayor transparencia y responsabilidad socio-económica de la gestión de las empresas e instituciones, sobre sus decisiones principales y, especialmente, en lo relativo a ofrecer una información relevante y continuada en el tiempo sobre los hechos más significativos que afectan a la eficiencia de la entidad, así como a su comportamiento relacional con los participes en suactividad económica o con los grupos de interés internos y externos de la organización. Las tecnologías de la información y las comunicaciones tienen un papel relevante en esta nueva estrategia de gobierno, al incorporar al gobierno de las empresas el uso de la tecnología de Internet que facilita la adopción de nuevas prácticas y la posibilidad de participación de todos los agentes sociales.

La weby el voto electrónico son herramientas cada vez más utilizadas por aquellas empresas que desean involucrar a sus accionistas en la vida social de la empresa. El voto electrónico se utiliza, de forma indistinta, para voto a distancia y voto delegado. Las empresas que utilizan el voto electrónico como forma de participación de los derechos sociales son empresas de alta capitalización, que tienen mucha visibilidad en el mercado y que desean que los inversores potenciales las conozcan y los accionistas actuales confíen en su actuación.

El uso de Internet en la población mundial tiene un ritmo ascendente, lo que obliga a las empresas a utilizar este medio para comunicarse con los inversores y permitir su participación activa. Pero en la actualidad, las empresas que más herramientas proporcionan a sus accionistas e inversores a través de Internet son aquellas de mayor capitalización, que poseen más recursos financieros, tecnológicos y personales para ejecutar proyectos de esta envergadura.

La aplicación de la tecnología puede ser una buena oportunidad para que los consejos de administración abran una vía de diálogo en la página web de la empresa, aprovechando algún hecho relevante que se desee comunicar. Así debería haber un canal directo de comunicación bajo la denominación "el consejo informa", por 
el cual pudieran los accionistas comunicarse con el consejo de administración y exponerles sus propuestas y dudas, y el consejo respondiera a ellas y explicara qué está haciendo la empresa para lograr sus objetivos. Para conseguir que los accionistas se sientan confiados y seguros con la relación que mantienen con la empresa, deben poder predecir qué va ocurrir en el futuro.

Pero para que estas formas de participación lleguen a desarrollarse plenamente es necesario que se desarrollen cambios importantes en la legislación como ya están produciéndose en la sociedad.

De nuevo, mientras la tecnología va adelantando, el derecho se va quedando atrás. Para que ambos vayan a la par es necesaria una adaptación del derecho mercantil existente actualmente en la mayoría de los países. Un campo con amplias posibilidades de aplicación a la vista de la globalización de capitales existente en la actualidad.

Cabe de más decir que este análisis abre camino para investigaciones futuras como la evolución del uso de las nuevas tecnologías para el gobierno de la empresa y los efectos que el uso de dichas tecnologías tiene para la empresa, el Consejo de la Administración y los accionistas, entre otras. 


\section{ReferenCiAs}

Alchian A. y Demsetz H. (1972). "Production, information cost and economic organization”. American Economic Review, Vol. 7, 3, pp. 266-287.

Alonso Almeida, M. M. (2007). El gobierno corporativo electrónico. Análisis desde el enfoque de confianza. Madrid. Editorial Netvision.

Backer, G.; Gibbons, R. y Murphy, K. J. (2002). "Relational contracts and the theory of the firm". Quartely Journal of Economics, 117, pp. 39-84.

Belanger, F. y Hiller, J. (2005). "A framework for e-government: Privacy implications". Business Process Management Journal, Vol. 11, pp. 57-81.

Bolton, G. E. y Katok, E., (2004). "How Effective Are Electronic Reputation Mechanisms? An Experimental Investigation". Management Science, Vol. 50, No. 11, pp. 1587-1602.

Bonsón, E. y Escobar, T. (2006). "Digital Reporting in Eastern Europe: An empirical studies". International Journal of Accounting Information Systems, Vol.7, pp. 299-318.

Bonsón, E. y Escobar, T. (2002). "A survey on voluntary disclosure on the Internet. Empirical evidence from 300 European Union companies". International Journal Digital Accounting Research, Vol.2, No.1, pp. 27-53.

Bonson, E.; Escobar, T. y Sánchez, M. (2000). Corporate digital reporting in Europe. A survey on Eurostoxx50 companies. Munich: 3rd European Conference in Accounting Information System.

Bueno Campos, E. (Ed.) (2004). El gobierno de la empresa. En busca de la transparencia y la confianza. Madrid: Editorial Pirámide.

Dahlberg, T., Mallat, N. y Oorni, A. (2003). Trust enhanced Technology Acceptance Model-Consumer acceptance of mobile payment solutions. Proceedings of the CIC Roundtable 2003. Disponible en: http://web.hhs.se/cic/roundtable2003/papers/D31_ Dahlberg_et_al.pdf

Davis, S. (1989). "Perceived usefulness, perceived ease of use, and user acceptance of Information Technology". MIS Quarterly, 319- 340. 
Fama, E.F. y Jensen, M.C. (1983). "Separation of ownership and control". The Journal of Law and Economics, 25, pp. 301-326.

Foss, N. J. (2005). Strategy and organization in knowledge economy. Oxford: Oxford University Press.

Gandía, J. L. y Andrés, T. (2005). E-Gobierno Corporativo y transparencia informativa en las sociedades cotizadas españolas: un estudio empírico. Monografía no 8 Dirección de Estudios y Estadísticas. Madrid: Comisión Nacional del Mercado de Valores.

Gowthorpe, C. y Amat, O. (1999). "External reporting of accounting and financial information via the Internet in Spain". European Accounting Review, Vol. 8, No. 2, pp. 365-371.

Grandori, A. (2004). Corporate governance and firm organization. Oxford: Oxford University Press.

Grant, R.M. (1996). Dirección Estratégica. Conceptos, Técnicas y Aplicaciones. Madrid: Civitas.

Grossman, S. y Hart, O. (1986). "An Analysis of the Principal-Agent Problem”. Econometrica, 51, pp. 7-45.

Handy, C. (1995). "Trust and virtual organization". Harvard Business Review, Vol.73, No. 3, pp. 40-50.

Hedlin, P. (1999). "The Internet as a vehicle for investor information; the Swedish case". European Accounting Review, Vol. 8, No. 2, pp. 373-381.

Hoffman, D.L. y Novak, T.P. (1996). "Marketing in Hypermedia Computer-Mediated Environments: Conceptual Foundations”. Journal of Marketing, Vol. 60, pp. 50-68.

Hossain, M.; Perera, H.B. y Rahman, A. R. (1995). "Voluntary disclosure in the annual reports of New Zealand companies". Journal International Finance $\mathcal{E}$ Management Accounting, 6, pp. 69-87.

Jensen, M.C. y Meckling, W.H. (1976). "Theory of the Firm: Managerial Behaviour, Agency Costs and Ownership Structure”. Journal of Financial Economics, 3, pp. 305-360.

Jones, S.J. (Ed.). (1995): Cyber Society. Computer-mediated communication and community. Thousand Oaks, CA: Sage.

Larrán, M. y Giner, B. (2002). "The use of the Internet for corporate reporting by Spanish companies". International Journal of Digital Accounting Research, Vol. 2, No.1, pp. 55-85. 
Lymer, A. (1998). The use of the Internet for corporate reporting. A discussion of the issues and survey of current usage in the UK. Antwerp, Belgium: 21st Annual Congress of the European Accounting Association.

Lymer, A. y Tallberg, A. (1997). Corporate reporting and the Internet - a survey and commentary on the use of the WWW in corporate reporting in the UK and Finland. Graz, Austria: 20th Annual Congress of the European Accounting Association.

Marston, C. L. y Polei, A. (2004). "Corporate reporting on the Internet by German companies". International Journal of Accounting Information System, Vol. 5, pp. 285-311.

Muñoz Paredes, J. M. (2004). Nuevas tecnologías en el funcionamiento de las Juntas generales y de los consejos de administración. Madrid: Editorial Civitas.

Nonaka, I. y Takeuchi, H. (1995). The knowledge-creating company. How Japanese companies create the dynamics of innovation. Nueva York: Oxford University Press.

Oyelere, P.; Laswad, F. y Fisher, R. (2003). "Determinants of Internet financial reporting by New Zealand companies". Journal of International Finance $\mathcal{E}$ Management Accounting, 14, (1), pp. 2663.

Pavlou, P.A. (2003). "Consumer acceptance of electronic commerce: Integrating trust and risk with the Technology Acceptance Model". International Journal of Electronic Commerce, Vol. 7, No. 3, 101-134.

Rajan, R. G. Y Zingales, L. (1998). "Power in a theory of the firm". Quarterly Journal Economics, 113, pp. 387-432.

Rodríguez Fernández, J. M. (2003). El gobierno de la empresa: un enfoque alternativo. Madrid: Ediciones Akal.

Rogers, E. M. (1996). Diffusion of Innovations. Nueva York: The Free Press.

Saudagaran, S. y Diga, G.K. (1997). "A review of research in the relationship between international capital markets and financial reporting by multinational firms". Journal of Accounting Literature, Vol. 16, pp. 127-159.

Spender, J. (1996). "Making knowledge the basis of a dinamic theory of the firm”. Strategic Management Journal, 17, pp. 45-62. 
Stevens, J. (1996). Applied Multivariate Statistics for the Social Sciences. Nueva Jersey: Lawrence Erlbaum.

Waltz, K. (1999). "Globalization and Governance". Political science and Politics, Vol. 32, No. 4, pp. 693-700.

Teece, D.J.; Pisano, G. y Shuen, A. (1997). "Dynamic capabilities and strategic management”. Strategic Management Journal, 18, (7), pp. 509-533.

Watts, R. y Zimmerman, J. (1978). "Towards a positive theory of the determination of accounting standards". Accounting Review, 53, pp. 112-134.

Wernerfelt, B. (1984). “A Resource Based View of the Firm”. Strategic Management Journal, 5, pp.171-180.

Williamson, O.E. (1985). The economic institutions of capitalism. Nueva York: Free Press.

Zingales, L. (2000). "In search of new foundations". Journal of Finance, 55, pp. 1623-1653. 